\title{
Obinutuzumab effective against treatment-refractory NHL
}

Despite improvements in the overall survival of patients with indolent non-Hodgkin lymphoma (NHL) over the past several decades, patients with rituximab-resistant disease have few other effective treatment options. Now researchers have demonstrated that the humanized anti-CD20 antibody obinutuzumab, in combination with bendamustine, is an effective treatment option in this patient population.

In a phase III, open-label, randomized controlled trial, 396 patients with CD20-positive, indolent NHL were randomized to receive either bendamustine monotherapy, or bendamustine plus obinutuzumab induction therapy for a total of six 28-day treatment cycles.

The use of obinutuzumab induction therapy resulted in significant improvements in patients' progression-free survival outcomes (median not reached at the time of publication), relative to those treated with bendamustine alone (median time to progression 14.9 months), despite similar numbers of patients in both groups having a response to treatment. Patients receiving obinutuzumab plus bendamustine also had a similar frequency of adverse events compared with those who received bendamustine alone, with neutropenia, thrombocytopenia and anaemia being the most commonly observed grade 3-4 adverse events in both groups. In total, 12 adverse event-related deaths were observed in both treatment groups: three of these deaths in the obinutuzumab plus bendamustine group, versus five in the bendamustine monotherapy group were considered treatment related.

These data indicate that obinutuzumab, which has a mechanism of action similar to that of rituximab, is an effective and safe treatment option in patients with rituximab-refractory NHL.

\section{Peter Sidaway}

ORIGINAL ARTICLE Sehn, L. H. et al. Obinutuzumab plus bendamustine versus bendamustine monotherapy in patients with rituximab-refractory indolent non-Hodgkin lymphoma (GADOLIN): a randomised, controlled, open-label, multicentre, phase 3 trial. Lancet Oncol. http://dx.doi.org/10.1016/S14702045(16)30097-3 (2016) 